\title{
Bone marrow fibrosis as a feature of systemic lupus erythematosus: a case report and literature review
}

Émilie Chalayer ${ }^{1 *}$, Martine Ffrench ${ }^{2}$ and Pascal Cathébras $^{1}$

\begin{abstract}
Introduction: Peripheral cytopenias are common in systemic lupus erythematosus (SLE), but bone marrow involvement is rarely reported. Myelofibrosis is a rare disorder characterized by reticulin fibrosis of the bone marrow, which usually occurs in response to clonal proliferation of hematopoietic stem cells in myeloproliferative disorders. However, bone marrow fibrosis has also been described in association with auto-immune diseases, especially SLE.

Method: We will report here a new case of bone marrow fibrosis associated with SLE. We also reviewed the 27 cases published in the English language literature, and will discuss the clinical presentation, outcome, treatment, and pathophysiology of bone marrow fibrosis occurring in association with SLE.

Results: Over one half of patients were diagnosed concomitantly with bone marrow fibrosis and SLE. Epidemiological, clinical and biological features of lupus were unremarkable. Except for the presence of reticulin fibrosis, the findings from the bone marrow biopsies proved highly variable. Overall mortality was about 14\% but corticosteroid-based therapy lead to clinical improvement and reverted bone marrow fibrosis in most cases. Data on the usefulness of other immunomodulatory therapies are inconclusive.

Conclusions: SLE may be complicated by bone marrow involvement, of a likely autoimmune origin. Bone marrow fibrosis occurring with SLE is probably similar to "primary autoimmune myelofibrosis" and may respond to steroid and immunomodulatory therapies. Further studies with standardised proofreading of bone marrow aspirations and biopsies are needed to delineate the clinical and biological features of this rare complication of SLE.
\end{abstract}

Keywords: Primary myelofibrosis; Bone marrow; Systemic lupus erythematosus

\section{Introduction}

Hematological abnormalities such as anemia, auto-immune hemolysis, leukopenia, lymphopenia and thrombocytopenia are very common in systemic lupus erythematosus (SLE) (Beyan et al. 2007). Blood cytopenias are among the criteria for SLE in the revised American College of Rheumatology (ACR) (Hochberg 1997) guidelines and may occur as the first symptom of the disease. Most of these manifestations are caused by increased peripheral destruction of blood cells associated with circulating auto-antibodies. Occasional case reports and small series have documented bone marrow abnormalities in patients with SLE, such as myelofibrosis,

\footnotetext{
* Correspondence: emilie.chalayer@chu-st-etienne.fr

${ }^{1}$ Service de médecine interne, Hôpital Nord, CHU de Saint-Étienne, 42055

Saint-Étienne, Cedex 2, France

Full list of author information is available at the end of the article
}

aplastic anemia, pure red cell aplasia, and features suggestive of myelodysplastic syndromes, suggesting that the bone marrow may also be a target organ in the disease. Bone marrow fibrosis is defined by the deposition of reticulin fibres in the bone marrow stroma. Fibrosis usually occurs in response to the clonal proliferation of hematopoietic stem cells (Kuter et al. 2007). Sporadic reports have suggested that bone marrow fibrosis may be part of the SLE disease spectrum, and the relationship between disease control and pancytopenia improvement provides indirect evidence for a causal relationship between SLE and bone marrow fibrosis. Autoimmune myelofibrosis may respond to immunosuppressive treatment with regression of the fibrosis and regeneration of the normal marrow tissue (Pullarkat et al. 2003). However, bone marrow involvement in SLE has not been thoroughly studied and the etiological mechanisms of this rare

\section{Springer}


complication remain unclear. We report here a case of bone marrow fibrosis associated with SLE. In order to improve the knowledge on this rare but serious complication of SLE, we have also reviewed all previously published cases.

\section{Case report}

A 17 year-old woman with a history of polyarthralgias and photosentivity was referred to the hospital in 1999 for the evaluation of a Raynaud's phenomenon. Clinical examination revealed no anomaly. She presented with neutropenia (leukocyte count $2.4 \times 10^{9} / 1$ with $1.1 \times 10^{9} / 1$ neutrophil granulocytes and $0.74 \times 10^{9} / 1$ lymphocytes) without anemia or thrombocytopenia. Antinuclear antibodies were present ( $>1 / 1280$ with a speckled fluorescence pattern). Anti-DNA antibodies were absent. Anti-U1-RNP and anti-SSA antibodies were positive. Three years later (2002), she was admitted to the hospital because of atypical eating disorder and psychotic behaviour. No evidence for neuropsychiatric lupus was found despite an extensive work-up. The patient was diagnosed with schizophrenia and treated with olanzapine. Nine years later (2011), she was referred to the hospital for fatigue, fever and pancytopenia. With the exception of extreme paleness, clinical examination was unremarkable. Her weight is normal. Laboratory findings on admission showed the following values: leukocyte count $0,48 \times 10^{9} / 1$ with $0.28 \times 10^{9} / 1$ neutrophil granulocytes and $0.08 \times 10^{9} / 1$ lymphocytes, hemoglobin $2.9 \mathrm{~g} / \mathrm{dl}$, platelet count $15 \times 10^{9} / \mathrm{l}$ and reticulocyte count $3 \times 10^{9} / 1$, low-normal level of folate, normal levels of vitamin B12 and ferritin. Anti-SSA, anti-RNP 70 and anti-U1-RNP antibodies were positive. The anti-DNA antibody titer was $76 \mathrm{UI} / \mathrm{ml}$ in ELISA [normal range $<10$ ]. Direct Coombs' test was negative. $\mathrm{C} 3, \mathrm{C} 4$, and $\mathrm{CH} 50$ were respectively $1.18 \mathrm{~g} / 1$ [normal range: 0.79-1.52], $0.12 \mathrm{~g} / \mathrm{l}$ [normal range: $0.2-0.51$ ] and $120 \%$. Abdominal ultrasonography showed limit homogen splenomegaly (around $12 \mathrm{~cm}$ ). Tear drop cells or leukoerythroblastic blood smear were not noted. Two attempts to aspirate bone marrow at different sites were unsuccessful and yielded only dry taps. Bone marrow biopsy showed hypercellular marrow (cellularity: 80\%) with focal lymphocytic infiltration, dysmyelopoiesis, erythrophagocytosis and grade 1-2 fibrosis. JAK-2, MPL W515L/K, and calreticulin mutations were not screen. A diagnosis of SLE with bone marrow involvement was retained. High dose methylprednisolone $(500 \mathrm{mg} / \mathrm{d})$ was given for 3 days. Then hydroxychloroquine and prednisone $(1 \mathrm{mg} / \mathrm{kg})$ were started, in addition to supportive care with blood transfusion and antibiotics. However, the patient remained pancytopenic. Intravenous immune globulins $(30 \mathrm{~g} / \mathrm{d})$ were given for 4 days with remarkable improvement. Hemoglobin level after 2 months was $11.8 \mathrm{~g} / \mathrm{dl}$, platelet count was $175 \times 10^{9} / \mathrm{l}$ and leucocyte count was $3.27 \times 10^{9} / 1$ with $2.32 \times 10^{9} / 1$ neutrophil granulocytes and $0.49 \times 10^{9} / 1$ lymphocytes Prednisone was continued for 17 months and gradually tapered. The patient did not experience change in her mental status during the course of steroid treatment. She remains in good physical health and still takes hydroxychloroquine and olanzapine.

\section{Review of published cases Method}

An electronic search of the literature was performed via MEDLINE by crossing the key words "systemic lupus erythematosus" AND ["bone marrow fibrosis" OR "myelofibrosis"]. We then examined additional references from the retrieved articles. The study period ran from January 1975 through December 2013. Only papers written in English were reviewed.

\section{Results}

\section{Clinical presentation (Table 1)}

Of the 27 cases retrieved from the English language literature and our case, 3 were males and 25 females, giving a ratio of 1:9. The age range was 12 to 70 years with a mean of 36 years and a median of 29 years. Fifteen patients received a concomitant diagnosis of SLE and bone marrow fibrosis, and 13 patients who had been previously diagnosed with SLE subsequently developed bone marrow fibrosis. In these 13 cases, the onset of bone marrow fibrosis varied from 8 months to 13 years after SLE diagnosis, with a mean of 5 years. Seven of these patients were under corticosteroids at the time of the hematological complication, two patients were under hydroxychloroquine, one had discontinued azathioprine at least 4 months before (Kiss et al. 2000) and one had started azathioprine two weeks before, but 4 weeks after discontinuing the drug no improvement had been observed (Vora et al. 1998). In patients with previously diagnosed SLE, symptoms attributed to the disease before bone marrow fibrosis occurred were rheumatologic symptoms (11/13), muco-cutaneous symptoms (8/13), renal involvement (3/13), serositis (3/13), and seizures (1/13). For all 28 patients, at the time of admission for cytopenias subsequently attributed to bone marrow fibrosis, the physical findings suggestive of SLE were rheumatologic symptoms $(8 / 28)$, renal involvement $(7 / 28)$, serositis $(6 / 28)$ and muco-cutaneous symptoms (4/28). Splenomegaly was found in $11 / 26$ patients ( 2 patients had undergone splenectomy due to immune thrombocytopenia), hepatomegaly in 11 patients, and lymph node enlargement or small diffuse lymph nodes in 6 patients. The other major symptoms reported were fever (17/28) and bleeding (15/28). Previous hematological history was often unclear, but at least 2 patients had been diagnosed with immune thrombocytopenia before the diagnosis of bone marrow fibrosis. 
Table 1 Clinical presentation

\begin{tabular}{|c|c|c|c|c|c|c|c|c|}
\hline $\mathrm{N}^{\text {ref }}$ & Age (yrs) & $M / F$ & PD SLE & SLE symptoms and signs & Associed symptoms and signs & LNE & HMG & SMG \\
\hline 1 (Sarkar et al. 2009) & 45 & M & - & Pleural effusion & Fever, melena, epistaxis & - & - & - \\
\hline 2 (Sacre et al. 2009) & 44 & $\mathrm{~F}$ & + & Diffuse alopecia, arthritis & & & & - \\
\hline 3 (Pillai et al. 2009) & 40 & $\mathrm{~F}$ & - & $\begin{array}{l}\text { Proteinuria, pleural and pericardial } \\
\text { effusion, ascites }\end{array}$ & Fever & - & + & - \\
\hline 4 (Aziz et al. 2004) & 22 & $\mathrm{~F}$ & - & Membranous nephropathy & Fever, epistaxis & + & + & + \\
\hline 5 (Kiss et al. 2000) & 18 & $\mathrm{~F}$ & + & Alopecia, facial erythema, arthritis & Fever, weight loss, myositis & & - & - \\
\hline 6 (Durupt et al. 2000) & 29 & $\mathrm{~F}$ & + & $\begin{array}{l}\text { Polyarthritis, mesangial } \\
\text { glomerulonephritis }\end{array}$ & Fever, hematuria & - & - & - \\
\hline 7 (Vora et al. 1998) & 22 & $\mathrm{~F}$ & + & Membranous nephropathy, seizures & Severe recurrent posterior scleritis & & & + \\
\hline $\begin{array}{l}8 \text { (Aharon et al. 1997; } \\
\text { Amital et al. 2003) }\end{array}$ & 54 & $\mathrm{~F}$ & - & Arthralgias, pleural effusion & Fever, weight loss, abdominal pain & + & + & + \\
\hline 9 (Agarwal et al. 1995) & 12 & $\mathrm{~F}$ & + & NS & Fever, petechias & - & - & - \\
\hline $\begin{array}{l}10 \text { (Ramakrishna et al. } \\
\text { 1995) }\end{array}$ & 18 & $\mathrm{~F}$ & - & Alopecia, arthralgias, Evans' syndrome & $\begin{array}{l}\text { Fever, weight loss, menorrhagia, } \\
\text { headaches }\end{array}$ & - & - & - \\
\hline 11 (Paquette et al. 1994) & 68 & M & - & Pleural effusion, oral ulcers & & & & - \\
\hline 12 (Paquette et al. 1994) & 27 & $\mathrm{~F}$ & - & NS & $\begin{array}{l}\text { Epistaxis, hematuria, gingival bleeding, } \\
\text { petechias }\end{array}$ & + & & + \\
\hline 13 (Paquette et al. 1994) & 23 & $\mathrm{~F}$ & + & $\begin{array}{l}\text { Photosensitivity, alopecia, malar rash, } \\
\text { pharyngeal ulcerations, arthritis, oral } \\
\text { ulcers }\end{array}$ & Gingival bleeding, retinal lesion & & + & + \\
\hline 14 (Paquette et al. 1994) & 56 & $\mathrm{~F}$ & + & Alopecia, arthritis & Cutaneous vasculitis & & & - \\
\hline 15 (Paquette et al. 1994) & 18 & $\mathrm{~F}$ & - & NS & $\begin{array}{l}\text { Fever, menorrhagia, epistaxis, gingival } \\
\text { bleeding, petechias }\end{array}$ & + & + & + \\
\hline 16 (Paquette et al. 1994) & 70 & $\mathrm{~F}$ & - & Arthritis, pleural effusion & Fever, weight loss, hematemesis & & & - \\
\hline 17 (Paquette et al. 1994) & 62 & $\mathrm{~F}$ & - & Psychosis & Fever, weight loss, panniculitis & & + & + \\
\hline 18 (Paquette et al. 1994) & 69 & $\mathrm{~F}$ & + & Arthritis, immune thrombocytopenia & Petechias & & + & S \\
\hline 19 (Hirose et al. 1993) & 54 & $\mathrm{~F}$ & + & Oral ulcers, polyarthralgias & Fever, weight loss & & & \\
\hline 20 (Borba et al. 1993) & 39 & $\mathrm{~F}$ & + & $\begin{array}{l}\text { Malar rash, photosentivity, alopecia, } \\
\text { Raynaud's, arthritis, pericarditis, } \\
\text { pleural effusion }\end{array}$ & Fever & & & \\
\hline $\begin{array}{l}21 \text { (Foley-Nolan et al. } \\
\text { 1992) }\end{array}$ & 20 & $\mathrm{~F}$ & - & Arthralgias & & & & - \\
\hline 22 (Inoue et al. 1992) & 24 & $\mathrm{~F}$ & - & Proteinuria & Fever, purpura & - & - & - \\
\hline 23 (Matsouka et al. 1989) & 60 & $\mathrm{~F}$ & - & $\begin{array}{l}\text { Proteinuria, immune } \\
\text { thrombocytopenia }\end{array}$ & $\begin{array}{l}\text { Fever, weight loss, petechias and } \\
\text { ecchymoses }\end{array}$ & & + & S \\
\hline 24 (el Mouzan et al. 1988) & 13 & $\mathrm{~F}$ & - & Arthritis & $\begin{array}{l}\text { Fever, anorexia, petechias and } \\
\text { ecchymoses }\end{array}$ & + & + & + \\
\hline $\begin{array}{l}25 \text { (Kaelin and Spivak } \\
\text { 1986) }\end{array}$ & 27 & $\mathrm{~F}$ & + & $\begin{array}{l}\text { Hyperpigmented skin rash, } \\
\text { polyarthralgias }\end{array}$ & $\begin{array}{l}\text { Ecchymoses, epistaxis, melena, gingival } \\
\text { bleeding, purpura }\end{array}$ & - & - & - \\
\hline 26 (Nanji and Jetha 1984) & 28 & M & + & $\begin{array}{l}\text { Erythematous macular lesions, arthritis, } \\
\text { deterioration in renal function, pleural } \\
\text { and pericardial effusion, ascites }\end{array}$ & Fever & & + & + \\
\hline 27 (Daly and Scott 1983) & 16 & $\mathrm{~F}$ & - & Alopecia, arthritis & $\begin{array}{l}\text { Weight loss, subcutaneous nodules in } \\
\text { hands, retinal exudates, epistaxis, gingival } \\
\text { bleeding, purpura and ecchymoses }\end{array}$ & + & + & + \\
\hline Present & 29 & $\mathrm{~F}$ & + & $\begin{array}{l}\text { Photosensitivity, polyarthralgias and } \\
\text { Raynaud's phenomenon }\end{array}$ & Fever, edema & - & - & + \\
\hline
\end{tabular}

PD: previous diagnosis of SLE, LNE: lymph node enlargement, HMG: hepatomegaly, SMG: splenomegaly, S: splenectomy, NS: not specified.

Peripheral hematological abnormalities (Table 2)

Thirteen of the 28 patients had pancytopenia (anemia: $\mathrm{Hb}<10 \mathrm{~g} / \mathrm{dl}$, leukopenia: $\mathrm{WBC}<4 \times 10^{9} / \mathrm{l}$, and thrombocytopenia: platelets $<150 \times 10^{9} / \mathrm{l}$ ), 13/28 had bicytopenia (anemia or/and leukopenia or/and thrombocytopenia), 1/28 had thrombocytopenia and neutropenia without leukopenia 
Table 2 Biology

\begin{tabular}{|c|c|c|c|c|c|c|c|c|c|c|c|c|c|}
\hline $\mathrm{N}^{\text {ref }}$ & Age yrs & $M / F$ & $\mathrm{Hb}$ g/dl & $\mathrm{PI} 10^{9} / \mathrm{I}$ & WBC $10^{9} / \mathrm{I}$ & $\mathrm{N} 10^{9} / \mathrm{I}$ & $\mathrm{L} 10^{9} / \mathrm{I}$ & ANA & DNA & other & Co test & LC & Bone marrow examination \\
\hline 1 (Sarkar et al. 2009) & 45 & M & 5.3 & 25 & 2.5 & 1.2 & 1 & $1 / 640$ & + & & + & & Hypercellular marrow with focal lymphocytic infiltration. \\
\hline 2 (Sacre et al. 2009) & 44 & $\mathrm{~F}$ & 7 & 65 & & & & high & + & & - & & Hypercellularity with marked reticulin fibrosis. \\
\hline 3 (Pillai et al. 2009) & 40 & $\mathrm{~F}$ & 10.6 & 25 & & 0 & & $1 / 320$ & - & & + & + & $\begin{array}{l}\text { Hypercellular with increased megakaryocytes } \\
\text { and reticulin (grade } 3 \text { ). }\end{array}$ \\
\hline 4 (Aziz et al. 2004) & 22 & $\mathrm{~F}$ & 6.2 & 18 & 1.2 & & & $1 / 160$ & & & & + & $\begin{array}{l}\text { Hypercellularity with an increased number of } \\
\text { megakaryocytes. Silver stain showed increased } \\
\text { reticulin fibrosis and the presence of patchy } \\
\text { areas of collagenized marrow. }\end{array}$ \\
\hline 5 (Kiss et al. 2000) & 18 & $\mathrm{~F}$ & 4.2 & 28 & 0.9 & & & high & & $\mathrm{aCL}$ & & & $\begin{array}{l}\text { Significant increase in the amount of reticulin } \\
\text { fibres ( } 40 \% \text {, Beumaister } 3-4) \text {, hypocellularity of } \\
\text { the myeloid components and massive } \\
\text { lymphocytic infiltration. }\end{array}$ \\
\hline 6 (Durupt et al. 2000) & 29 & $\mathrm{~F}$ & 9.4 & 102 & 1.1 & 0.6 & 0.3 & $1 / 1120$ & + & & & + & $\begin{array}{l}\text { All normal hematopoietic elements with a small } \\
\text { increase in mature megakaryocytes and marked } \\
\text { inflammatory medullar reaction with plasmocytosis, } \\
\text { systematized increase in reticulin (grade 1). }\end{array}$ \\
\hline 7 (Vora et al. 1998) & 22 & $\mathrm{~F}$ & 9 & 60 & 4 & & & $1 / 1000$ & - & & & & $\begin{array}{l}\text { Marked osteomyelosclerosis with severe fibrosis } \\
\text { of the marrow spaces and near-total effacement } \\
\text { of normal hematopoiesis. }\end{array}$ \\
\hline $\begin{array}{l}8 \text { (Aharon et al. 1997; } \\
\text { Amital et al. 2003) }\end{array}$ & 54 & $\mathrm{~F}$ & 5.1 & 96 & 3.1 & 2.2 & 0.6 & high & + & $\begin{array}{l}\mathrm{aCL}, \text { anti-histone, } \\
\text { anti-SSA }\end{array}$ & & + & $\begin{array}{l}\text { Focal hypercellularity, many reticulin and collagen } \\
\text { fibers, normal appearance of the red cell and white } \\
\text { cell lines, mild megakaryocytosis with few mildly } \\
\text { abnormal megakaryocytes. }\end{array}$ \\
\hline 9 (Agarwal et al. 1995) & 12 & $\mathrm{~F}$ & 9.6 & 4 & 4.7 & 0.7 & 3.3 & $1 / 40$ & - & & - & & $\begin{array}{l}\text { Extremely hypocellular marrow with } \\
\text { residual patches of hematopoietic cells. Reticulin } \\
\text { stain showed a marked increase in fine fibrosis, } \\
\text { which was diffuse grade } 3 \text {. }\end{array}$ \\
\hline 10 (Ramakrishna et al. 1995) & 18 & $\mathrm{~F}$ & 5.4 & 30 & 6 & & & $1 / 2560$ & + & $\begin{array}{l}\mathrm{LA}, \mathrm{aCL}, \\
\text { antiplatelet }\end{array}$ & + & + & $\begin{array}{l}\text { Markedly hypercellular marrow with erythroid } \\
\text { hyperplasia, plentiful megakaryocytes and } \\
\text { markedly increased reticulin. }\end{array}$ \\
\hline 11 (Paquette et al. 1994) & 68 & M & 5.7 & 222 & 3.5 & & & $1 / 5280$ & - & & & + & $\begin{array}{l}\text { Hypercellular, increased stroma and reticulin } \\
\text { fibrosis. }\end{array}$ \\
\hline 12 (Paquette et al. 1994) & 27 & $\mathrm{~F}$ & 5 & 1 & 5.9 & & & $1 / 80$ & - & & + & - & Fibrosis with megakaryocyte clustering. \\
\hline 13 (Paquette et al. 1994) & 23 & $\mathrm{~F}$ & 9.5 & 55 & 4.2 & & & high & & & & - & Fibrosis, open sinusoids, megakaryocyte clusters. \\
\hline 14 (Paquette et al. 1994) & 56 & $\mathrm{~F}$ & 9.7 & 76 & 5.4 & & & high & + & & - & + & $\begin{array}{l}\text { Erythroid hyperplasia, increased megakaryocytes, } \\
\text { fibroblast proliferation, reticulin fibrosis. }\end{array}$ \\
\hline 15 (Paquette et al. 1994) & 18 & $\mathrm{~F}$ & 2.7 & 4 & 7.7 & & & high & + & & + & + & $\begin{array}{l}\text { Bone marrow fibrosis, hypercellular marrow, } \\
\text { increased megakaryocytes with clustering. }\end{array}$ \\
\hline 16 (Paquette et al. 1994) & 70 & $\mathrm{~F}$ & 4.2 & 20 & 3.8 & & & high & - & & + & + & $\begin{array}{l}\text { Advanced bone marrow fibrosis, hypocellular } \\
\text { marrow, predominance of megakaryocytes. }\end{array}$ \\
\hline 17 (Paquette et al. 1994) & 62 & $\mathrm{~F}$ & 9.3 & 35 & 3 & & & high & - & & & + & $\begin{array}{l}\text { Advanced bone marrow fibrosis, marked } \\
\text { osteosclerosis. }\end{array}$ \\
\hline
\end{tabular}


Table 2 Biology (Continued)

\begin{tabular}{|c|c|c|c|c|c|c|c|c|c|c|c|c|c|}
\hline 18 (Paquette et al. 1994) & 69 & $\mathrm{~F}$ & 6.8 & 39 & 6.8 & & & high & - & & & - & $\begin{array}{l}\text { Hypercellular with increased, clustering } \\
\text { megakaryocytes; stroma moderately } \\
\text { increased by accumulation of fibrillar } \\
\text { reticulin. }\end{array}$ \\
\hline 19 (Hirose et al. 1993) & 54 & $F$ & 6.9 & 17 & 1.7 & & & $1 / 320$ & - & $\mathrm{aCL}, \mathrm{LA}$ & & + & $\begin{array}{l}\text { Marked marrow fibrosis with reduced numbers } \\
\text { of erythroid precursors and normal proliferation } \\
\text { of both megakaryocytes and myeloid series, } \\
\text { the reticulin content was increased. }\end{array}$ \\
\hline 20 (Borba et al. 1993) & 39 & $\mathrm{~F}$ & 9.7 & 341 & 2.4 & 0.9 & & $1 / 200$ & - & anti-neutrophil & + & + & $\begin{array}{l}\text { All normal hematopoeitic elements with } \\
\text { localised fibrosis, reticulin was moderately } \\
\text { increased. }\end{array}$ \\
\hline 21 (Foley-Nolan et al. 1992) & 20 & $\mathrm{~F}$ & 10.5 & 45 & 4.5 & 1.3 & 1.9 & $1 / 800$ & & & + & + & $\begin{array}{l}\text { Hypercellular with a marked increase in the } \\
\text { number of megakaryocytes and a diffuse, } \\
\text { significant increase in reticulin content. }\end{array}$ \\
\hline 22 (Inoue et al. 1992) & 24 & $F$ & 6.8 & 2 & 3.8 & & & $1 / 100$ & - & antiplatelet & - & + & $\begin{array}{l}\text { Bone marrow fibrosis characterized by marked } \\
\text { hyperplasic marrow with an increase in reticulin } \\
\text { fibers, all elements including megakaryocytes } \\
\text { increased in number. }\end{array}$ \\
\hline 23 (Matsouka et al. 1989) & 60 & $F$ & & 20 & 4 & 1.4 & 1.4 & $1 / 320$ & & & - & - & $\begin{array}{l}\text { Presence of dense fibrous tissue with fibroblasts, } \\
\text { paucity of immature and polymorphonuclear cells. }\end{array}$ \\
\hline 24 (el Mouzan et al. 1988) & 13 & $\mathrm{~F}$ & 6.5 & 10 & 1.3 & 0.2 & 1 & $1 / 640$ & + & rhumatoid factor & + & + & $\begin{array}{l}\text { Decreased bone marrow activity with 1:1 myeloid } \\
\text { to erythroid ratio, megakaryocytes quantitatively } \\
\text { normal, increased reticulo-endothelial activity } \\
\text { and fibrosis. }\end{array}$ \\
\hline 25 (Kaelin and Spivak 1986) & 27 & $\mathrm{~F}$ & 13.1 & 5 & 4.4 & 2.8 & 0.9 & $1 / 1280$ & + & $\begin{array}{l}\text { antiplatelet, } \\
\text { anti-neutrophil }\end{array}$ & + & + & $\begin{array}{l}\text { Contained all normal hematopoietic elements } \\
\text { with a slight increase in mature megakaryocytes, } \\
\text { markedly increased reticulin. }\end{array}$ \\
\hline 26 (Nanji and Jetha 1984) & 28 & M & 8.4 & 1 & 0.3 & & & high & & & - & & $\begin{array}{l}\text { Hypocellular in some areas and hypercellular in } \\
\text { others, megakaryocytes decreased in number, } \\
\text { increased numbers of histiocytes and fibroblasts, } \\
\text { extensive fibrosis and diffuse increased in reticulin. }\end{array}$ \\
\hline 27 (Daly and Scott 1983) & 16 & $F$ & 7 & 28 & 1.7 & 0.5 & & $1 / 1280$ & + & & - & + & $\begin{array}{l}\text { Marked marrow fibrosis with reduced numbers of } \\
\text { erythroid precursors, plentiful megakaryocytes, } \\
\text { greatly increased reticulin content. }\end{array}$ \\
\hline Present & 29 & $F$ & 2.9 & 15 & 0.48 & 0.28 & 0.08 & $1 / 1280$ & + & $\begin{array}{l}\text { anti-SSA, RNP 70, } \\
\text { U1-RNP }\end{array}$ & - & + & $\begin{array}{l}\text { Hypercellular marrow (cellularity : } 80 \% \text { ) with focal } \\
\text { lymphocytic infiltration, dysmyelopoiesis, } \\
\text { erythrophagocytosis and grade } 1-2 \text { fibrosis. }\end{array}$ \\
\hline
\end{tabular}


and $1 / 28$, only thrombocytopenia. Neutropenia $(<1,5 \times$ $10^{9} / \mathrm{l}$ ) was observed in 10/12 (16 missing data) patients, lymphopenia in 4/9 (19 missing data), hemolysis with hyper-reticulocytosis in one case and a positive direct Coombs' test in 10/18 (10 missing data). Hemoglobin levels ranged from 13.1 to $2.7 \mathrm{~g} / \mathrm{dl}$ (mean $7.3 \mathrm{~g} / \mathrm{dl}$ ), platelets from 341 to $1 \times 10^{9} / 1$ (mean $50 \times 10^{9} / \mathrm{l}$ ), and leucocytes from 6.8 to $0.35 \times 10^{9} / 1$ (mean $\left.3.4 \times 10^{9} / \mathrm{l}\right)$. Of the 24 patients with thrombocytopenia, 11 had deep $\left(<20 \times 10^{9} / \mathrm{l}\right)$, 8 severe $\left(<50 \times 10^{9} / 1\right)$, and 5 moderate thrombocytopenia. Tear drop cells or leukoerythroblastic blood smear, two common finding in primary myelofibrosis, were noted in 14 patients.

\section{Bone marrow abnormalities (Table 2)}

For 22/28 patients, a "dry tap" occurred during bone marrow aspiration. All bone marrow biopsies showed bone marrow fibrosis with variable increases in the amount of reticulin fibers and fibroblasts. Grades of bone marrow fibrosis were not always specified. Global marrow cellularity was variable, ranging from increased $(12 / 28)$ to normal $(11 / 28)$ or decreased $(5 / 28)$ without any increase in blasts. All elements including megakaryocytes appeared morphologically normal. Megakaryocytes were increased or/and with clustering in 13/28 patients and decreased in $1 / 28$. Focal or massive lymphocytic infiltration was observed in $4 / 28$, plasmocytosis in $1 / 28$ and erythroid hyperplasia in $2 / 28$. Fifteen patients underwent repeated bone marrow examination showing improvement, with reduction in reticulin amounts in $12 / 15$.

\section{Immunological abnormalities (Table 2)}

Antinuclear antibodies were found in all patients, antidsDNA in $12 / 28$, anti-Ro/SSA in $2 / 28$, anti-histone in $1 / 28$, and a low complement level in $18 / 28$ patients. Antiphospholipid antibodies were detected in $4 / 28$ patients. Antiplatelet antibodies testing came out positive in 3 patients and negative in 4 .

\section{Outcome and treatment (Table 3)}

Follow-up time ranged from a few months to years but was often unspecified. The overall mortality was $14 \%(4 / 28)$. Two patients died within few days with no other treatment than blood transfusions and antibiotics. Two other patients who died received only prednisone as a specific treatment. Improvement was noted in 17/ 28 patients, transient response with need for new treatment in $5 / 28$, and no improvement in $2 / 28$. Supportive care with antibiotics and transfusions was explicitly mentioned for 8 patients. Two patients received granulocyte colony stimulating factor (G-CSF).

Immunomodulatory therapies consisted in corticosteroids (26/28) (prednisone, prednisolone or methylprednisolone), intravenous immune globulins (4/28) (Ramakrishna et al. 1995; Aharon et al. 1997; Sacre et al. 2009), plasma exchanges (2/28) (Borba et al. 1993; Vora et al. 1998), azathioprine (2/28) (Foley-Nolan et al. 1992; Kiss et al. 2000), cyclophosphamide (1/28) (Borba et al. 1993), cyclosporine (1/28) (Kiss et al. 2000), danazol (1/28) (Ramakrishna et al. 1995), colchicine (1/28) (Ramakrishna et al. 1995), vincristine (1/28) (Ramakrishna et al. 1995) and splenectomy (1/28) (Ramakrishna et al. 1995). Of 20 patients who received only corticosteroids, 16 improved and 4 did not. Four patients who received cortisone concomitantly with azathioprine, intravenous immunoglobulin or cyclosporine improved. One patient received cortisone and showed a transient response but pancytopenia relapsed so she received danazol, vincristine, colchicine, intravenous immunoglobulins, then underwent splenectomy and finally improved (Ramakrishna et al. 1995). Another patient was treated with plasma exchanges and cyclophosphamide following a transient response to cortisone and improved (Borba et al. 1993). One patient received intravenous immune globulins after 3 weeks of corticosteroid treatment without response, and a marked improvement occurred within the following week (Aharon et al. 1997).

\section{Discussion \\ Nosology}

Primary myelofibrosis is considered as a clonal myeloproliferative disorder (Tefferi et al. 2012). However some diseases such as infections, neoplasms and autoimmune diseases may also induce bone marrow fibrosis. The term "myelofibrosis" is used in some contexts to describe any increase in bone marrow stromal fibres, regardless of the associated disease, and in other contexts to define a specific myeloid disorder (primary myelofibrosis) (Kuter et al. 2007). The word "myelofibrosis" is therefore ambiguous, and in this article we have chosen rather to use the term "bone marrow fibrosis". Some authors suggest the importance of distinguishing between increases in bone marrow reticulin and collagen. Abovenormal reticulin amounts are generally regarded as a nonspecific sign of bone marrow abnormality, but may or may not be a sign of serious neoplastic disease. In contrast, increased collagen is less common and is mainly seen in tumours metastatic to the bone marrow or in the late stages of myeloproliferative diseases. Unlike increased reticulin, it is not always reversible (Kuter et al. 2007). In most cases reported here, it was unclear if trichrome collagen stain and/or reticulin stain were performed, and the type and amount of fibrosis were not reported according to established grading scales (Kuter et al. 2007). Another issue is whether finding bone marrow reticulin fibrosis per se should prompt a diagnosis of autoimmune myelofibrosis in a patient with SLE. For example, mild degrees of reticulin fibrosis can be 
Table 3 Treatment

\begin{tabular}{|c|c|c|c|c|c|}
\hline$\overline{\mathrm{N}^{\text {ref }}}$ & Age (yrs) & $\operatorname{Sex}(M / F)$ & $\begin{array}{l}\text { Medication received before } \\
\text { bone marrow fibrosis }\end{array}$ & $\begin{array}{l}\text { Immunomodulatory therapy } \\
\text { for bone marrow fibrosis }\end{array}$ & Response \\
\hline 1 (Sarkar et al. 2009) & 45 & M & & Prednisolone $60 \mathrm{mg} / \mathrm{d}$ & Improved \\
\hline 2 (Sacre et al. 2009) & 44 & $\mathrm{~F}$ & Prednisone, hydroxychloroquine & Prednisone $1 \mathrm{mg} / \mathrm{kg} / \mathrm{d}$, IVlg & Improved \\
\hline 3 (Pillai et al. 2009) & 40 & $\mathrm{~F}$ & & $\begin{array}{l}\text { IV methylprednisolone } 500 \mathrm{mg} / \mathrm{d} \text {, } \\
\text { prednisone }\end{array}$ & Improved \\
\hline 4 (Aziz et al. 2004) & 22 & $\mathrm{~F}$ & & Prednisone $1 \mathrm{mg} / \mathrm{kg} / \mathrm{d}$ & Improved \\
\hline 5 (Kiss et al. 2000) & 18 & $\mathrm{~F}$ & $\begin{array}{l}\text { Prednisone, azathioprine } \\
\text { discontinued } 6 \text { months prior }\end{array}$ & $\begin{array}{l}\text { IV methylprednisolone } 1 \mathrm{~g} / \mathrm{d} \times 3 \text {, } \\
\text { prednisone } 2 \mathrm{mg} / \mathrm{kg} / \mathrm{d} \text {, cyclosporine } \\
3 \mathrm{mg} / \mathrm{kg} \text {, azathioprine }\end{array}$ & $\begin{array}{l}\text { Relapsed, secondary } \\
\text { improvement }\end{array}$ \\
\hline 6 (Durupt et al. 2000) & 29 & $\mathrm{~F}$ & & Prednisone $2 \mathrm{mg} / \mathrm{kg} / \mathrm{d}$ & Improved \\
\hline 7 (Vora et al. 1998) & 22 & $\mathrm{~F}$ & $\begin{array}{l}\text { Prednisone, azathioprine initiated } \\
2 \text { weeks prior }\end{array}$ & $\begin{array}{l}\text { Azathioprine discontinuation, } 6 \text { plasma } \\
\text { exchanges, IV methylprednisolone } \\
1 \mathrm{~g} \text { pulses }\end{array}$ & Improved \\
\hline $\begin{array}{l}8 \text { (Aharon et al. 1997; Amital } \\
\text { et al. 2003) }\end{array}$ & 54 & $\mathrm{~F}$ & & $\begin{array}{l}\text { Prednisone } 80 \mathrm{mg} / \mathrm{d} \text {, IVlg } \\
400 \mathrm{mg} / \mathrm{kg} / \mathrm{d} \times 5\end{array}$ & Improved \\
\hline 9 (Agarwal et al. 1995) & 12 & $\mathrm{~F}$ & & Prednisone $2 \mathrm{mg} / \mathrm{kg} / \mathrm{d}$ & Improved \\
\hline 10 (Ramakrishna et al. 1995) & 18 & $\mathrm{~F}$ & & $\begin{array}{l}\text { Prednisolone } 75 \mathrm{mg} / \mathrm{d} \text {, IVlg, splenectomy, } \\
\text { danazol, colchicine, vincristine }\end{array}$ & $\begin{array}{l}\text { Relapsed, secondary } \\
\text { improvement }\end{array}$ \\
\hline 11 (Paquette et al. 1994) & 68 & M & & Prednisone 20 mg/d & Not improved \\
\hline 12 (Paquette et al. 1994) & 27 & $\mathrm{~F}$ & & Prednisone $60 \mathrm{mg} / \mathrm{d}$ & Improved \\
\hline 13 (Paquette et al. 1994) & 23 & $\mathrm{~F}$ & & Prednisone $50 \mathrm{mg} / \mathrm{d}$ & Improved \\
\hline 14 (Paquette et al. 1994) & 56 & $\mathrm{~F}$ & Prednisone & Prednisone & Not improved \\
\hline 15 (Paquette et al. 1994) & 18 & $\mathrm{~F}$ & & Prednisone $80 \mathrm{mg} / \mathrm{d}$ & Improved \\
\hline 16 (Paquette et al. 1994) & 70 & $\mathrm{~F}$ & & Prednisone & Deceased \\
\hline 17 (Paquette et al. 1994) & 62 & $\mathrm{~F}$ & & & Deceased \\
\hline 18 (Paquette et al. 1994) & 69 & $\mathrm{~F}$ & & Prednisone, splenectomy & $\begin{array}{l}\text { Relapsed, secondary } \\
\text { improvement }\end{array}$ \\
\hline 19 (Hirose et al. 1993) & 54 & $\mathrm{~F}$ & & $\begin{array}{l}\text { IV methylprednisolone } 1 \mathrm{~g} / \mathrm{d} \times 3 \text {, } \\
\text { prednisone } 60 \mathrm{mg} / \mathrm{d}\end{array}$ & Improved \\
\hline 20 (Borba et al. 1993) & 39 & $\mathrm{~F}$ & Prednisone, hydroxychloroquine & $\begin{array}{l}\text { Methylprednisolone, prednisone, } \\
\text { plasma exchanges, cyclophosphamide }\end{array}$ & $\begin{array}{l}\text { Relapsed, secondary } \\
\text { Improvement }\end{array}$ \\
\hline 21 (Foley-Nolan et al. 1992) & 20 & $\mathrm{~F}$ & & $\begin{array}{l}\text { Prednisolone } 40 \mathrm{mg} / \mathrm{d} \text {, azathioprine } \\
50 \mathrm{mg} / \mathrm{d}\end{array}$ & $\begin{array}{l}\text { Relapsed, secondary } \\
\text { improvement }\end{array}$ \\
\hline 22 (Inoue et al. 1992) & 24 & $\mathrm{~F}$ & & $\begin{array}{l}\text { Prednisolone } 1,2 \mathrm{mg} / \mathrm{kg} / \mathrm{d} \\
\text { IV methylprednisolone } 1 \mathrm{~g} / \mathrm{d} \times 3\end{array}$ & Improved \\
\hline 23 (Matsouka et al. 1989) & 60 & $\mathrm{~F}$ & & Hydrocortisone $1 \mathrm{~g} / \mathrm{d}$ & Deceased \\
\hline 24 (el Mouzan et al. 1988) & 13 & $\mathrm{~F}$ & & Prednisolone $30 \mathrm{mg} / \mathrm{d}$ & Improved \\
\hline 25 (Kaelin and Spivak 1986) & 27 & $\mathrm{~F}$ & Prednisone, salicyclate & $\begin{array}{l}\text { IV methylprednisolone } 100 \mathrm{mg} / \mathrm{d} \times 6 \text {, } \\
\text { prednisone } 50 \mathrm{mg} / \mathrm{d}\end{array}$ & Improved \\
\hline 26 (Nanji and Jetha 1984) & 28 & M & Prednisone & & Deceased \\
\hline 27 (Daly and Scott 1983) & 16 & $\mathrm{~F}$ & $\begin{array}{l}\text { NSAID including } \\
\text { oxyphenbutazone }\end{array}$ & Prednisolone $30 \mathrm{mg} / \mathrm{d}$ & Improved \\
\hline Present & 29 & $\mathrm{~F}$ & Olanzapine & $\begin{array}{l}\text { IV methylprednisolone } 500 \mathrm{mg} / \mathrm{d} \text { ×3, } \\
\text { prednisone } 1 \mathrm{mg} / \mathrm{kg} \text { and } \\
\text { hydroxychloroquine, IVlg } 30 \mathrm{~g} / \mathrm{d} \times 4\end{array}$ & Improved \\
\hline
\end{tabular}

NSAID: non-steroidal anti-inflammatory drugs. IVIg: intravenous immunoglobulins.

observed in conditions such as immune thrombocytopenia and may be found in many patients with lupus when routine bone marrow biopsies are performed (Pereira et al. 1998). Moreover, some authors have reported cases of bone marrow fibrosis in patients who do not have SLE or other well-defined autoimmune syndromes (Bass et al. 2001; Pullarkat et al. 2003). They have defined "primary autoimmune myelofibrosis" as a disorder characterized by 
cytopenias with bone marrow lymphocyte infiltration and grade $3-4$ reticulin fibrosis of the bone marrow, lack of atypical bone marrow cells or osteosclerosis, absent or mild splenomegaly, and the presence of auto-antibodies. In our review, the 28 retrieved cases have been considered as fulfilling criteria for SLE, although lupus symptoms and signs leading to the diagnosis of SLE were not always reported in detail by the authors. Their clinico-pathological features were very similar to those of the reported cases of "primary autoimmune myelofibrosis". Thus we tend to believe that "autoimmune myelofibrosis", just like autoimmune cytopenias, may occur as an isolated disorder, or as a feature of other autoimmune diseases including SLE. Finally, cases of aplastic anemia have also been reported in SLE patients. We found 25 published cases in the English language literature (Aplastic anemia as a feature of systemic lupus erythematosus. In preparation). In these cases, the bone marrow biopsy showed marked hypocellularity, but the absence of reticulin fibrosis was often not specified, and thus the differentiation between "lupus bone marrow fibrosis" and "lupus aplastic anemia" is not always clear, raising the question of the borderland between these two rare features of SLE (Cavalcant et al. 1978).

\section{Pathophysiology}

The pathogenesis of bone marrow fibrosis remains incompletely understood, but appears to be a relatively nonspecific response of fibroblasts to underlying cellular abnormalities. Increased reticulin is the result of fibroblast proliferation, and increased collagen synthesis or altered collagen turnover appear to be due to decreased collagenase release from macrophages and neutrophils (Kuter et al. 2007). Several growth factors appear to be implicated. The platelet-derived growth factor (PDGF), found in megakaryocytes and platelets, stimulates fibroblast growth (Kuter et al. 2007). The transforming growth factor $\beta$ (TGF $\beta$ ) and epidermal growth factor (EGF) are known to promote collagen synthesis (Le Bousse-Kerdilès et al. 2008). Immunological abnormalities may be involved in the pathogenesis. The increased circulating immune complexes and auto-antibodies that are present in SLE may act on megakaryocyte Fc receptors and release growth factors to promote marrow fibrosis. Some authors have suggested that both auto-antibodies against CD34+ stem cells and cytotoxic $\mathrm{T}$ cells may initiate and perpetuate damage to the bone marrow (Kiss et al. 2000). An increase in leucocyte apoptosis and impaired clearance of apoptotic cells has also been observed in patients with SLE. These apoptotic bodies were observed in the bone marrow of patients with SLE, while they are not typically seen in normal bone marrow. Delayed apoptotic cell clearance leads to prolonged exposure of auto-antigens and predisposes to antibody production (Hepburn et al. 2007). Furthermore, in the bone marrow of patients with bone marrow fibrosis and SLE, megakaryocyte counts are often above normal or normal. Therefore thrombocytopenia may result at least partly from an increased destruction of the platelets rather than a decreased production caused by bone marrow fibrosis. An association between immune thrombocytopenia (to which bone marrow dysfunction is increasingly believed to contribute (Gernsheimer 2009)) and bone marrow fibrosis has been observed in 3 of the 28 reported cases.

The JAK2 V617F mutation, associated with primary myeloproliferative disorders, is present in up to one half of the patients with primary myelofibrosis (Tefferi et al. 2012). Some authors suggest a thorough search for auto-immunity in the absence of the mutation (Sacre et al. 2009).

\section{Clinical and biological presentation}

Primary myelofibrosis is diagnosed relatively late in life (median age is 66 years) and is more common in males (ratio 3:2) (Tefferi et al. 2012). Bone marrow fibrosis occurring with SLE is diagnosed earlier (median age is 29 years) and is very uncommon in males (ratio 1:9). In $15 / 28$ cases, the diagnosis of SLE and bone marrow fibrosis were made simultaneously. However, in 5 of these cases a number of symptoms and signs (such as arthralgias, alopecia, proteinuria) were suggestive of undiagnosed yet pre-existing SLE (Daly and Scott 1983; Matsouka et al. 1989; Inoue et al. 1992; Paquette et al. 1994; Pillai et al. 2009). Some authors suggest that autoimmune disorders, including SLE, may be considered in cases of bone marrow fibrosis in patients whose spleen is not enlarged (Pullarkat et al. 2003; Sacre et al. 2009), but 10/26 patients in our review had splenomegaly. Moreover, in primary myelofibrosis, the clinical finding of splenomegaly is associated with collagen, but not reticulin fibrosis (Thiele and Kvasnicka 2006). Fifteen patients underwent repeated bone marrow examinations showing improvement, with a reduction in reticulin in $12 / 15$. This suggests that reticulin fibrosis (and maybe even collagen fibrosis) can be reversed if the underlying disease is treated (Pereira et al. 1998).

\section{Outcome and treatment}

Although it is likely that negative outcomes are less frequently reported in case reports, for which follow up data may be lacking, and that consequently the overall mortality may be higher than the $14 \%$ documented from this review, this mortality rate suggests a more favourable course for SLE-associated bone marrow fibrosis than for primary myelofibrosis (Tefferi et al. 2012). Interestingly, bone marrow fibrosis occurring with SLE appears to often respond to corticosteroids, unlike primary myelofibrosis. Plasma exchanges seem to have no efficacy. Intravenous immune globulins were used for 4 patients only (Ramakrishna et al. 1995; Aharon et al. 1997; 
Sacre et al. 2009), and proved to be efficient in at least one patient (Aharon et al. 1997), as it was the case for the patient we managed. None of the 28 patients received rituximab. None received an allogeneic hematopoietic stem cell transplant.

\section{Conclusion}

SLE may be complicated by bone marrow fibrosis, which is likely to be of autoimmune origin. This feature may be more common than previously thought, with cases being incorrectly characterized as blood peripheral cytopenias in patients previously diagnosed with SLE, and cases being misdiagnosed with primary myelofibrosis in patients not previously diagnosed with SLE. We think that in patients with SLE, cytopenias should be confirmed by bone marrow aspiration, and by bone marrow biopsy in atypical or refractory cases. Moreover, autoimmune myelofibrosis or SLE-associated bone marrow fibrosis should be considered in cases of primary myelofibrosis with atypical features such as young age and female sex, absence of spleen enlargement, or absence of JAK2 V617F mutation, because this condition seems amenable to efficient treatment. High-dose corticosteroid therapy with or without intravenous immune globulins should be the first-line therapy.

In order to improve knowledge of bone marrow involvement in SLE, we have established a French registry, with centralized proofreading of bone marrow aspirations and biopsies. We hope to achieve a sufficient sample size for epidemiological and clinical research on this unusual feature of lupus.

\section{Consent}

Written informed consent was obtained from the patient for the publication of this report.

\section{Competing interests}

The authors declare no conflict of interests.

\section{Authors' contributions}

All authors participated and drafted the manuscript. All authors read and approved the final manuscript.

\footnotetext{
Author details

${ }^{1}$ Service de médecine interne, Hôpital Nord, CHU de Saint-Étienne, 42055 Saint-Étienne, Cedex 2, France. '2Laboratoire d'hématologie, Centre Hospitalier Lyon Sud, 165 Chemin du Grand Revoyet, 69495 Pierre Bénite, France.

Received: 20 June 2014 Accepted: 26 June 2014

Published: 9 July 2014

\section{References}

Agarwal BR, Bhalla K, Dalvi R, Currimbhoy ZE, Mehta KP (1995) Myelofibrosis secondary to SLE and its reversal on steroid therapy. Indian Pediatr 32:1207-1210 Aharon A, Levy Y, Bar-Dayan Y, Afek A, Zandman-Goddard G, Skurnik Y, Fabrrizzi F, Shoenfeld $Y$ (1997) Successful treatment of early secondary myelofibrosis in SLE with IVIG. Lupus 6:408-411, doi:10.1177/096120339700600412

Amital H, Rewald E, Levy Y, Bar-Dayan Y, Manthorpe R, Engervall P, Sherer Y, Langevitz P, Shoenfeld Y (2003) Fibrosis regression induced by intravenous gammaglobulin treatment. Ann Rheum Dis 62:175-177. doi:10.1136/ard.62.2.175
}

Aziz AR, Ashraf R, Mohammadian Y, Ruby C, Momin Z, Kumar A, Griciene P, Gintautas J (2004) Systemic lupus erythematosus presenting with pancytopenia due to bone marrow myelofibrosis in a 22-year-old male. Clin Adv Hematol Oncol 2:467-469, discussion 469-470

Bass RD, Randall D, Pullarkat V, Feinstein DI, Kaul A, Winberg CD, Brynes RK (2001) Pathology of autoimmune myelofibrosis a report of three cases and a review of the literature. Am J Clin Pathol 116:211-216

Beyan E, Beyan C, Turan M (2007) Hematological presentation in systemic lupus erythematosus and its relationship with disease activity. Hematology 12:257-261, doi:10.1080/10245330701214145

Borba EF, Pereira RM, Velloso ED, Pereira IA, Goncalves CR, Yoshinari NH (1993) Neutropenia associated with myelofibrosis in systemic lupus erythematosus. Acta Haematol 89:82-85

Cavalcant J, Shadduck RK, Winkeistein A, Zeigler Z, Mendelow H (1978) Red-cell hypoplasia and increased bone marrow reticulin in systemic lupus erythematosis: Reversal with corticosteroid therapy. Am J Hematol 5:253-263

Daly HM, Scott GL (1983) Myelofibrosis as a cause of pancytopenia in systemic lupus erythematosus. J Clin Pathol 36:1219-1222, doi:10.1136/jcp.36.11.1219

Durupt S, David G, Durieu I, Nove-Josserand R, Vital DD (2000) Myelofibrosis in systemic lupus erythematosus: a new case. Eur J Intern Med 11:98-100

El Mouzan Ml, Ahmad MA, al Fadel Saleh M, al Sohaibani MO, al Gindan YM (1988) Myelofibrosis and pancytopenia in systemic lupus erythematosus. Acta Haematol 80:219-221

Foley-Nolan D, Martin MF, Rowbotham D, McVerry A, Gooi HC (1992) Systemic lupus erythematosus presenting with myelofibrosis. J Rheumatol 19:1303-1304

Gernsheimer T (2009) Chronic idiopathic thrombocytopenic purpura: mechanisms of pathogenesis. Oncologist 14:12-21, doi:10.1634/theoncologist.2008-0132

Hepburn AL, Lampert IA, Boyle JJ, Horncastle D, Fai Ng W, Layton M, Vyse TJ, Botto M, Mason JC (2007) In vivo evidence for apoptosis in the bone marrow in systemic lupus erythematosus. Ann Rheum Dis 66:1106-1109, doi:10.1136/ard.2006.065003

Hirose W, Fukuya H, Anzai T, Kawagoe M, Kawai T, Watanabe K (1993) Myelofibrosis and systemic lupus erythematosus. J Rheumatol 20:2164-2166

Hochberg MC (1997) Updating the American College of Rheumatology revised criteria for the classification of systemic lupus erythematosus. Arthritis Rheum 40:1725-1734, doi:10.1002/1529-0131(199709)40:9<1725::AID-ART29>3.0.CO;2-Y

Inoue Y, Matsubara A, Okuya S, Okafuji K, Kaku K, Kaneko T (1992) Myelofibrosis and systemic lupus erythematosus: reversal of fibrosis with high-dose corticosteroid therapy. Acta Haematol 88:32-36

Kaelin WG, Spivak JL (1986) Systemic lupus erythematosus and myelofibrosis. Am J Med 81:935-938

Kiss E, Gaal IA, Simkovics EO, Kiss A (2000) Myelofibrosis in systemic lupus erythematosus. Leuk Lymphoma 39:661-665

Kuter DJ, Bain B, Mufti G, Bagg A, Hasserjian RP (2007) Bone marrow fibrosis: pathophysiology and clinical significance of increased bone marrow stromal fibres. Br J Haematol 139:351-362

Le Bousse-Kerdilès M-C, Martyré M-C, Samson M (2008) Cellular and molecular mechanisms underlying bone marrow and liver fibrosis: a review. Eur Cytokine Netw 19:69-80

Matsouka C, Liouris J, Andrianakos A, Papademetriou C, Karvountzis G (1989) Systemic lupus erythematosus and myelofibrosis. Clin Rheumatol 8:402-407

Nanji AA, Jetha N (1984) Myelofibrosis as a cause of pancytopenia in systemic lupus erythematosus. J Clin Pathol 37:714. doi:10.1136/jcp.37.6.714-a

Paquette RL, Meshkinpour A, Rosen PJ (1994) Autoimmune myelofibrosis. A steroid-responsive cause of bone marrow fibrosis associated with systemic lupus erythematosus. Medicine (Baltimore) 73:145-152

Pereira RMR, Velloso ERP, Menezes Y, Gualandro S, Vassalo J, Yoshinari NH (1998) Bone marrow findings in systemic lupus erythematosus patients with peripheral cytopenias. Clin Rheumatol 17:219-222

Pillai A, Gautam M, Williamson H, Martlew V, Nash J, Thachil J (2009) Multisystem failure due to three coexisting autoimmune diseases. Intern Med 48:837-842

Pullarkat V, Bass RD, Gong JZ, Feinstein DI, Brynes RK (2003) Primary autoimmune myelofibrosis: Definition of a distinct clinicopathologic syndrome. Am J Hematol 72:8-12, doi:10.1002/ajh.10258

Ramakrishna R, Kyle PW, Day PJ, Manoharan A (1995) Evans' syndrome, myelofibrosis and systemic lupus erythematosus: role of procollagens in myelofibrosis. Pathology 27:255-259

Sacre K, Aguilar C, Deligny C, Choudat L, Koch P, Arfi S, Papo T (2009) Lytic bone lesions in lupus-associated myelofibrosis. Lupus 19:313-316, doi:10.1177/ 0961203309349118 
Sarkar RN, Banerjee S, Dey S, Saha A, Bhattacharjee P, Banerjee TK, Sinha PK, Chakraborty A (2009) Haematological presentation of systemic lupus erythematosus. J Assoc Physicians India 57:767-768

Tefferi A, Lasho TL, Jimma T, Finke CM, Gangat N, Vaidya R, Begna KH (2012) One Thousand Patients With Primary Myelofibrosis: The Mayo Clinic Experience. Mayo Clin Proc 87:25-33, doi:10.1016/.mayocp.2011.11.001

Thiele J, Kvasnicka HM (2006) Grade of bone marrow fibrosis is associated with relevant hematological findings-a clinicopathological study on 865 patients with chronic idiopathic myelofibrosis. Ann Hematol 85:226-232, doi:10.1007/ s00277-005-0042-8

Vora BJ, Byers RJ, Lucas GS, Gokal R (1998) Reversal of osteomyelosclerosis-associated systemic lupus nephritis. Nephrol Dial Transplant 13:1559-1561, doi:10.1093/ndt/ 13.6 .1559

doi:10.1186/2193-1801-3-349

Cite this article as: Chalayer et al:: Bone marrow fibrosis as a feature of systemic lupus erythematosus: a case report and literature review.

SpringerPlus 2014 3:349.

\section{Submit your manuscript to a SpringerOpen ${ }^{\circ}$} journal and benefit from:

- Convenient online submission

- Rigorous peer review

- Immediate publication on acceptance

- Open access: articles freely available online

- High visibility within the field

- Retaining the copyright to your article

Submit your next manuscript at $\gg$ springeropen.com 